\title{
Mixing properties of induced random transformations
}

\author{
Hans-Otto Georgii \\ Mathematisches Institut der Universität München \\ Theresienstr. 39, D-80333 München \\ E-mail: georgii@rz.mathematik.uni-muenchen.de
}

\begin{abstract}
Let $S(N)$ be a random walk on a countable abelian group $G$ which acts on a probability space $E$ by measure-preserving transformations $\left(T_{v}\right)_{v \in G}$. For any $\Lambda \subset E$ we consider the random return time $\tau$ at which $T_{S(\tau)} \in \Lambda$. We show that the corresponding induced skew product transformation is $\mathrm{K}$-mixing whenever a natural subgroup of $G$ acts ergodically on $E$.
\end{abstract}

Key words: Random group action; skew product; induced transformation; Ksystem; random walk in a random landscape.

\section{Introduction and result}

This note is concerned with an abstract version of the following

Basic example: Kasteleyn's random walk in a random scenery. Let $\mathrm{Z}^{d}$ be the integer lattice of dimension $d \geq 1$, and suppose we are given

(i) a stochastic coloring of $\mathbf{Z}^{d}$, that is, a translation-invariant $\{0,1\}$-valued random field $C=(C(v))_{v \in \mathrm{Z}^{d}}$ - the vertices $v \in \mathrm{Z}^{d}$ with $C(v)=1$ will be called black; and

(ii) a random walk $\left(S_{N}\right)_{N \geq 0}$ on $Z^{d}$ which starts at the origin and is independent of the coloring.

Let $C_{0}=\left(C_{0}(v)\right)_{v \in \mathrm{Z}^{d}}$ be the conditioned coloring for which the origin is black, and let $\tau_{k}$ denote the $k$-th time at which the random walk visits a black point of $C_{0}$. (Note that the consideration of these times connects the two independent data, the coloring and the walk.) At time $\tau_{k}$, the random walker observes the coloring $C_{k}=\left(C_{0}\left(S_{\tau_{k}}+v\right)\right)_{v \in \mathrm{Z}^{d}}$ around his position. The conditioning of the coloring ensures that the sequence $\left(C_{k}\right)_{k \geq 0}$ of observed colorings is stationary. By Kakutani's random ergodic theorem [4], the process $\left(C_{k}\right)_{k \geq 0}$ is ergodic when the coloring $C$ is ergodic with respect to translations. Are there any stronger mixing properties of $\left(C_{k}\right)_{k \geq 0}$ which can be deduced from suitable mixing properties of $C$ ?

This question was posed by Kasteleyn in [5] - this paper gives a survey of the early work on the model above. A first answer was found by Keane and den Hollander [6]: If $C$ is a Bernoulli coloring and $\left(S_{N}\right)$ is transient, $\left(C_{k}\right)_{k \geq 0}$ is (strongly) mixing. This 
result was much improved by den Hollander [2]: $\left(C_{k}\right)_{k \geq 0}$ is mixing whenever $C$ has a trivial tail $\sigma$-field. He conjectured that the mixing property of $\left(C_{k}\right)_{k \geq 0}$ already holds when the coloring is ergodic with respect to the subgroup of translations generated by the differences of possible steps of the random walk. In this note we shall establish this conjecture. In fact, we will even show that (under the above assumption of ergodicity) the stationary process $\left(C_{k}\right)_{k \geq 0}$ has a trivial tail. Also, there will be no difficulty in considering a more general setting which we will describe now.

The setting. We consider the following objects:

- a standard Borel probability space $(E, \mathcal{B}, \mu)$;

- a countable abelian (additive) group $G$;

- a $\mu$-preserving measurable action $\left(T_{v}\right)_{v \in G}$ of $G$ on $E$ (which means by definition that the $T_{v}$ are $\mu$-preserving invertible transformations of $(E, \mathcal{B})$ such that $T_{v} T_{v^{\prime}}=T_{v+v^{\prime}}$ for $v, v^{\prime} \in G$ and $T_{0}=$ id for the null element 0 of $G$ );

- a probability measure $p$ on $G$ which serves as the jump distribution for a random walk on $G$. The latter is defined on the Bernoulli space $(W, \mathcal{W}, \nu)=(G, \mathcal{P}(G), p)^{\mathrm{N}}$ by $S_{N}(w) \equiv S(N, w)=\sum_{n=1}^{N} w_{n}$, where $w_{n}$ is the $n$-th coordinate of $w \in W$.

The joint system is described by the product space

$$
(\Omega, \mathcal{F}, P)=(E \times W, \mathcal{B} \otimes \mathcal{W}, \mu \otimes \nu)
$$

together with the skew product transformation

$$
T(x, w)=\left(T_{w_{1}} x, \theta w\right), \quad(x, w) \in \Omega,
$$

where $\theta: W \rightarrow W$ is the left-shift. It is well-known and easy to see that $T$ preserves $P$. Its iterates are given by the formula

$$
T^{N}(x, w)=\left(T_{S(N, w)} x, \theta^{N} w\right), \quad(x, w) \in \Omega, N \geq 1 .
$$

This shows that the first coordinates of $T^{N}(x, w)$ perform a random walk through the $G$-orbit of $x$.

We make the following assumption of ergodicity.

(A) $\mu$ is ergodic with respect to $\left(T_{v}\right)_{v \in \Gamma}$, where $\Gamma=\Gamma(p)$ is the smallest group containing

$$
\operatorname{supp} p-\operatorname{supp} p=\{u-v: u, v \in G, p(u) p(v)>0\} .
$$

$p$ is called strongly aperiodic if $\Gamma=G$. In this case assumption (A) simply means that $\mu$ is ergodic with respect to the whole transformation group $\left(T_{v}\right)_{v \in G}$.

The following proposition is essentially due to Meilijson [8] and den Hollander [2]. It can be phrased by saying that - due to the Bernoulliness of the random walk - the skew product transformation $T$ has much better mixing properties than the original transformation group $\left(T_{v}\right)_{v \in G}$.

Proposition Under assumption (A), $T$ is a Kolmogorov-endomorphism. That is, for all $A \in \mathcal{F}$ we have

$$
\sup _{B \in \mathcal{F}}\left|P\left(A \cap T^{-N} B\right)-P(A) P(B)\right| \rightarrow 0
$$


as $N \rightarrow \infty$.

Let us return for a moment to the basic example. In this case $G=Z^{d}, E=\{0,1\}^{G}$, $\mu$ is the distribution of the coloring, and $T_{v}$ the shift of $E$ by $v \in G$. The objects of study are the distinguished event $\Lambda=\{C(0)=1\}$ that the origin is black, and the sequence of random times $N$ at which $T_{S(N)} \in \Lambda$.

Abstracting again from the example, we thus need to include a final ingredient into our general setting, namely

- a fixed measurable set $\Lambda \subset E$ with $\mu(\Lambda)>0$.

For $(x, w) \in \Omega$ we consider the time

$$
\tau(x, w)=\inf \left\{N \geq 1: T_{S(N, w)} x \in \Lambda\right\}
$$

of the first visit in (or, if $x \in \Lambda$, first return to) $\Lambda$. The sequence of all later return times to $\Lambda$ is defined by the recursion $\tau_{1}=\tau, \tau_{k+1}=\tau_{k}+\tau \circ T^{\tau_{k}}$ for $k \geq 1$. As $T$ is ergodic, the Poincaré recurrence theorem implies that all $\tau_{k}$ are finite with $P$-probability one.

For a point $(x, w) \in \Lambda \times W$, the consideration of the return times $\tau_{k}$ amounts to considering the induced dynamical system $\left(\Lambda \times W, \mathcal{F}_{\Lambda}, P_{\Lambda}, T_{\Lambda}\right)$, where $\mathcal{F}_{\Lambda}$ is the restriction of $\mathcal{F}$ to $\Lambda \times W, P_{\Lambda}=P(\cdot \mid \Lambda \times W)=\mu(\cdot \mid \Lambda) \otimes \nu$ the conditional probability measure, and $T_{\Lambda}=T^{\tau}$ the induced transformation of $T$ on $\Lambda \times W$. Explicitly, for $(x, w) \in \Lambda \times W$ and $k \geq 0$ we have

$$
T_{\Lambda}^{k}(x, w)=\left(T_{S\left(\tau_{k}(x, w), w\right)} x, \theta^{\tau_{k}(x, w)} w\right) .
$$

Let us mention at this point that the central concepts of this paper — random group action and induced transformation - were both developed by Kakutani [3, 4].

What are the mixing properties of the induced dynamical system? In general, an induced transformation inherits only ergodicity, but no stronger mixing property from its primitive transformation. In fact, the stationary Markov chain with state space $\{0,1,2\}$ and transition probabilities $p_{00}=p_{01}=1 / 2, p_{12}=p_{20}=1$ provides an example of a K- (even Bernoulli) system for which the induced subchain with state space $\{1,2\}$ is periodic and therefore not weakly mixing. However, the dynamical system $(\Omega, \mathcal{F}, \mu, T)$ under consideration is so well-behaved that even the induced subsystem is $\mathrm{K}$-mixing. This is the essence of our main result.

Theorem Under assumption (A), the induced transformation $T_{\Lambda}$ is a Kolmogorovendomorphism. Explicitly, for all $A \in \mathcal{F}_{\Lambda}$,

$$
\sup _{B \in \mathcal{F}_{\Lambda}}\left|P_{\Lambda}\left(A \cap T_{\Lambda}^{-k} B\right)-P_{\Lambda}(A) P_{\Lambda}(B)\right| \rightarrow 0
$$

as $k \rightarrow \infty$.

Remarks (a) Setting $\Lambda=E$ in the theorem we reobtain the proposition. Nevertheless, for the sake of exposition we will first prove the proposition and then refine the argument to obtain the theorem. 
(b) As is well-known, (2) is equivalent to the statement that $P_{\Lambda}$ is trivial on the future tail $\sigma$-algebra $\bigcap_{k \geq 1} T_{\Lambda}^{-k} \mathcal{F}_{\Lambda}$. Another way of writing (2) is the following statement of convergence to equilibrium: For each $A \in \mathcal{F}_{\Lambda}$,

$$
\left\|P\left(T^{\tau_{k}} \in \cdot \mid A\right)-P_{\Lambda}\right\| \rightarrow 0
$$

as $k \rightarrow \infty$, where $\|\cdot\|$ stands for the total variation norm. Assertion (3) even holds when $A$ is not contained in $\Lambda$. Indeed, for each $A \in \mathcal{F}$ we have that $P\left(T^{\tau} \in \cdot \mid A\right) \ll P_{\Lambda}$, and a standard extension argument allows to insert the associated Radon-Nikodym density in the place of $A$ in (2). In fact, our proof of the theorem gives (3) directly for all $A \in \mathcal{F}$.

(c) Reducing (3) to events in $E$ we obtain: For all $\Delta \in \mathcal{B}$,

$$
\sup _{B \in \mathcal{B}}\left|\int \nu(d w) \mu\left(x: T_{S\left(\tau_{k}(x, w), w\right)} x \in B \mid \Delta\right)-\mu(B \mid \Lambda)\right| \rightarrow 0
$$

as $k \rightarrow \infty$. Another corollary of (3) is that for all $A \in \mathcal{F}$,

$$
\left\|P\left(\tau_{k+1}-\tau_{k} \in \cdot \mid A\right)-P_{\Lambda}(\tau \in \cdot)\right\| \rightarrow 0
$$

as $k \rightarrow \infty$. In particular, if $\int \tau d P<\infty$ then

$$
\int\left(\tau_{k+1}-\tau_{k}\right) d P(\cdot \mid A) \rightarrow \int \tau d P_{\Lambda}=1 / \mu(\Lambda)
$$

as $k \rightarrow \infty$ because in this case the sequence $\left(\tau_{k+1}-\tau_{k}\right)_{k \geq 1}$ is uniformly $P$-integrable. To see this one may use the equations $P(\tau=n)=\mu(\Lambda) P_{\Lambda}(\tau \geq n)$ and $P\left(\tau_{k+1}-\tau_{k}=\right.$ $n)=\mu(\Lambda) P_{\Lambda}\left(\tau ; \tau_{k+1}-\tau_{k}=n\right)$ for $n, k \geq 1$ which follow readily from the fact that $T$ is $P$-preserving and ergodic.

(d) The theorem breaks down for induced transformations $T_{M}$ on subsets $M$ of $\Omega$ which are not of the particular form $M=\Lambda \times W$. Here is a counterexample. Let $G=\mathrm{Z}$ and $p$ be such that $p(0)=p(1)=1 / 2$. Define $M=E \times\left\{w \in W: w_{1}=1\right\}$, and let $\tau_{k}$ denote the $k$-th return time to $M$. (Note that $\tau_{k}$ is a function of $w$ only.) Then $S\left(\tau_{k}\right)=k$ and $T_{M}^{k}=T_{k} \times \theta^{\tau_{k}}$. As a consequence, assertion (2) (with $T_{\Lambda}$ replaced by $T_{M}$ ) can only hold when $\mu$ is a Dirac measure. This shows that assumption (A) is by far not sufficient for $T_{M}$ to be $\mathrm{K}$-mixing.

(e) $T$ and $T_{\Lambda}$ become invertible if we replace $W$ by the two-sided sequence space $G^{\mathrm{Z}}$. It is routine to restate and prove the theorem in this setting.

We shall prove the proposition in Section 2 and the theorem in Section 3. As den Hollander in [2], we make extensive use of coupling arguments. Actually we adopt some of his ideas. The main difference is the following. Den Hollander used the tail triviality of the coloring to obtain a tail coupling of conditioned colorings. By this we mean a realization of the conditioned colorings on a common probability space which is such that the colorings coincide outside of a finite random box. He then had to deal with the difficulty that - in the case that the random walk is recurrent — the random box of disagreement will be visited infinitely many often. We can avoid this problem by replacing the tail coupling with an orbit coupling. This only requires the ergodicity of the coloring and provides a realization of conditioned colorings on a common probability space which is such that each coloring is a random translate of the other. This leads to great simplifications of the argument. 


\section{Proof of the proposition}

We start with stating the orbit coupling lemma which is our fundamental tool.

Lemma 2.1 (Orbit coupling) Suppose assumption (A) holds, and let $\Delta, \Delta^{\prime} \subset E$ be two measurable sets with $\mu(\Delta) \mu\left(\Delta^{\prime}\right)>0$. Then there exist a probability measure $\bar{\mu}=\bar{\mu}_{\Delta, \Delta^{\prime}}$ on $(E \times E, \mathcal{B} \otimes \mathcal{B})$ and a measurable function $\gamma: E \times E \rightarrow \Gamma$ such that

(i) $\bar{\mu}$ has marginals $\mu(\cdot \mid \Delta)$ and $\mu\left(\cdot \mid \Delta^{\prime}\right)$, and

(ii) $\bar{\mu}\left(X^{\prime}=T_{\gamma} X\right)=1$, where $X, X^{\prime}$ are the two projections from $E \times E$ onto $E$.

Proof. In view of (A), $\mu$ is trivial on the $\sigma$-algebra $\mathcal{I}(\Gamma)$ of all $\left(T_{v}\right)_{v \in \Gamma^{-}}$-invariant events in $E$. Hence $\mu(\cdot \mid \Delta)=\mu\left(\cdot \mid \Delta^{\prime}\right)$ on $\mathcal{I}(\Gamma)$. The lemma is therefore a particular case of the orbit coupling theorem (in fact of Proposition 3.1) in [1].

Our second tool is a coupling of random walks. It is merely a slight variant of a coupling proposed by Liggett on pp. 69-70 of [7]. For later use, however, we need to give the details of the proof.

Lemma 2.2 (Random walk coupling) Let $v \in \Gamma$ and $C, C^{\prime}$ two cylinder events in $W$ with $\nu(C) \nu\left(C^{\prime}\right)>0$. Then there exists a probability measure $\bar{\nu}=\bar{\nu}_{v, C, C^{\prime}}$ on $(W \times W, \mathcal{W} \otimes \mathcal{W})$ such that

(i) $\bar{\nu}$ has marginals $\nu(\cdot \mid C)$ and $\nu\left(\cdot \mid C^{\prime}\right)$, and

(ii) $\bar{\nu}\left(S_{N}=S_{N}^{\prime}+v\right.$ eventually $)=1$.

Here $S_{N}=S_{N}(\xi)$ and $S_{N}^{\prime}=S_{N}\left(\xi^{\prime}\right)$ for the two projections $\xi, \xi^{\prime}$ from $W \times W$ onto $W$.

Proof. We may assume that, for some $\ell \in \mathrm{N}, C$ and $C^{\prime}$ prescribe fixed values for the first $\ell$ coordinates. The general case then follows by an averaging argument.

For such $C, C^{\prime}$, the conditional probabilities $\nu(\cdot \mid C)$ and $\nu\left(\cdot \mid C^{\prime}\right)$ govern two random walks which start at time $\ell$ at certain points $u, u^{\prime} \in G$ with $u-u^{\prime} \in \Gamma$. This shows that we can, in fact, assume without loss that $C=C^{\prime}=W$.

By the definition of $\Gamma$, there exist some $k \in \mathrm{N}$ and $u_{1}, \ldots, u_{k}, u_{1}^{\prime}, \ldots, u_{k}^{\prime} \in \operatorname{supp} p$ such that $u_{j} \neq u_{j}^{\prime}$ for $1 \leq j \leq k$ and $\sum_{j=1}^{k}\left(u_{j}-u_{j}^{\prime}\right)=v$. We introduce the stopping times $\rho_{0}=0$ and

$$
\rho_{j}=\inf \left\{N>\rho_{j-1}: S_{N}-S_{N}^{\prime}=\sum_{i=1}^{j}\left(u_{i}-u_{i}^{\prime}\right)\right\},
$$

$1 \leq j \leq k$. We define a probability measure $\bar{\nu}$ on $W \times W=(G \times G)^{\mathrm{N}}$ by prescribing its recursive conditional probabilities. Namely, for $n \in \mathrm{N}$ and $1 \leq j \leq k$ we stipulate that on the set $\left\{\rho_{j-1}<n \leq \rho_{j}\right\}$,

$$
\bar{\nu}\left(\xi_{n}=a, \xi_{n}^{\prime}=a^{\prime} \mid\left(\xi_{i}, \xi_{i}^{\prime}\right), i<n\right)=\left\{\begin{array}{cl}
p(a) & \text { if } a=a^{\prime} \notin\left\{u_{j}, u_{j}^{\prime}\right\} \\
p(a)-\delta_{j} & \text { if } a=a^{\prime} \in\left\{u_{j}, u_{j}^{\prime}\right\} \\
\delta_{j} & \text { if }\left\{a, a^{\prime}\right\}=\left\{u_{j}, u_{j}^{\prime}\right\} \\
0 & \text { otherwise }
\end{array}\right.
$$


with $\delta_{j}=p\left(u_{j}\right) p\left(u_{j}^{\prime}\right)>0$, while on $\left\{\rho_{k}<n\right\}$ this conditional probability is equal to $p(a)$ if $a=a^{\prime}$ and 0 otherwise; here $a, a^{\prime} \in G$. [Note that the preceding definition makes sense because $\rho_{1}, \ldots, \rho_{k}$ are stopping times, so that the events $\left\{\rho_{j-1}<n \leq \rho_{j}\right\}$ are measurable with respect to $\sigma\left(\left(\xi_{i}, \xi_{i}^{\prime}\right), i<n\right)$.] Intuitively, the preceding prescription means that on the time interval $\left.] \rho_{j-1}, \rho_{j}\right]$ the two random walks $S_{N}$ and $S_{N}^{\prime}+v$ are allowed to make different steps of size $u_{j}$ or $u_{j}^{\prime}$, but otherwise run in unison. They also run in unison after the final coupling time $\rho_{k}$.

Since the preceding conditional probabilities on $G \times G$ have marginals $p$ independently of the condition, $\bar{\nu}$ satisfies (i). To prove (ii) we only need to show that $\bar{\nu}\left(\rho_{k}<\infty\right)=1$. This will follow once we have shown that the set $\left\{\rho_{j-1}<\infty=\rho_{j}\right\}$ has $\bar{\nu}$-probability zero for each $1 \leq j \leq k$. On this set, the sequence

$$
\left(S_{N}-S_{N}^{\prime}-\sum_{i=1}^{j-1}\left(u_{i}-u_{i}^{\prime}\right): N \geq \rho_{j-1}\right)
$$

performs a simple symmetric random walk on the one dimensional group $\left(u_{j}-u_{j}^{\prime}\right) \mathrm{Z}$ with neighbor transition probability $\delta_{j}$ and holding probability $1-2 \delta_{j}$, but never hits the point $\left(u_{j}-u_{j}^{\prime}\right)$. Since this cannot occur with positive probability, the proof of the lemma is complete.

We are now able to prove the proposition. The proof of the theorem will be quite similar but uses a refined coupling of random walks.

To establish (1) we can assume without loss that $A=\Delta \times C$ for some $\Delta \in \mathcal{B}$ and cylinder event $C \subset W$ such that $P(A)=\mu(\Delta) \nu(C)>0$. We choose $\bar{\mu}=\bar{\mu}_{\Delta, E}$ according to the orbit coupling lemma, and for $v \in \Gamma$ we let $\bar{\nu}_{v}=\bar{\nu}_{v, C, W}$ be as in Lemma 2.2. We define a probability measure $\bar{P}$ on $\Omega \times \Omega=E \times E \times W \times W$ by

$$
\bar{P}\left(d x, d x^{\prime}, d w, d w^{\prime}\right)=\bar{\mu}\left(d x, d x^{\prime}\right) \bar{\nu}_{\gamma\left(x, x^{\prime}\right)}\left(d w, d w^{\prime}\right) .
$$

Writing $X, X^{\prime}: \Omega \times \Omega \rightarrow E$ and $\xi, \xi^{\prime}: \Omega \times \Omega \rightarrow W$ for the four projections we then have $\bar{P}((X, \xi) \in \cdot)=P(\cdot \mid A)$ and $\bar{P}\left(\left(X^{\prime}, \xi^{\prime}\right) \in \cdot\right)=P$.

Consider the coupling time

$$
\rho=\inf \left\{M \geq 1: S_{N}=S_{N}^{\prime}+\gamma\left(X, X^{\prime}\right) \text { for all } N \geq M\right\} .
$$

By construction, $\bar{P}(\rho<\infty)=1$. On the set $\{\rho \leq N\}$ we have $\xi_{n}=\xi_{n}^{\prime}$ for all $n>N$ and

$$
T_{S(N)} X=T_{S^{\prime}(N)+\gamma\left(X, X^{\prime}\right)} X=T_{S^{\prime}(N)} X^{\prime} .
$$

This means that $T^{N}(X, \xi)=T^{N}\left(X^{\prime}, \xi^{\prime}\right)$ on $\{\rho \leq N\}$. Thus for each $B \in \mathcal{F}$ we can write, using that $P\left(T^{-1} B\right)=P(B)$,

$$
\begin{aligned}
\left|P\left(T^{-N} B \mid A\right)-P(B)\right| & \leq \int\left|1_{B} \circ T^{N}(X, \xi)-1_{B} \circ T^{N}\left(X^{\prime}, \xi^{\prime}\right)\right| d \bar{P} \\
& \leq \bar{P}(\rho>N) .
\end{aligned}
$$

Assertion (1) now follows immediately. 


\section{Proof of the theorem}

To prove (2) we can assume without loss that $\mu\left(\Lambda \triangle T_{v} \Lambda\right)>0$ for some $v \in \Gamma$. Indeed, in the alternative case $\Lambda$ is almost surely invariant under the transformation group $\left(T_{v}\right)_{v \in \Gamma}$. Assumption (A) then implies that $\mu(\Lambda)=1$, and this means that $P_{\Lambda}=P$ and $P\left(T_{\Lambda}=T\right)=1$. So in this case the theorem is reduced to the proposition which has already been proved.

Since $\left\{v \in \Gamma: \mu\left(\Lambda \triangle T_{v} \Lambda\right)=0\right\}$ is a group and $\Gamma$ is generated by $\operatorname{supp} p-\operatorname{supp} p$, the assumption above can be written in the form

(B) $\mu\left(T_{u} \Lambda \triangle T_{v} \Lambda\right)>0$ for some $u, v \in \operatorname{supp} p$.

We will use this property to introduce a refined coupling of random walks which accounts for the number of visits in $\Lambda$. Let

$$
V_{N}=\sum_{n=1}^{N} 1_{\Lambda \times W} \circ T^{n}=\sum_{n=1}^{N} 1_{\Lambda} \circ T_{S(n, \cdot)}
$$

be the number of visits in $\Lambda$ up to time $N$.

Lemma 3.1 Suppose (A) and (B) hold, and let $v \in \Gamma, k \in \mathrm{Z}$ and $C, C^{\prime}$ be two cylinder events with $\nu(C) \nu\left(C^{\prime}\right)>0$. Then there exists a probability kernel $x \rightarrow \bar{\nu}_{x}=\bar{\nu}_{x, v, k, C, C^{\prime}}$ from $(E, \mathcal{B})$ to $(W \times W, \mathcal{W} \otimes \mathcal{W})$ such that

(i) for all $x, \bar{\nu}_{x}$ has marginals $\nu(\cdot \mid C)$ and $\nu\left(\cdot \mid C^{\prime}\right)$, and

(ii) for $\mu$-almost all $x$,

$$
\bar{\nu}_{x}\left(S_{N}=S_{N}^{\prime}+v \text { and } V_{N}(x, \cdot)=V_{N}^{\prime}\left(T_{v} x, \cdot\right)+k \text { eventually }\right)=1 .
$$

Here $V_{N}(x, \cdot)=V_{N}(x, \xi)$ and $V_{N}^{\prime}(x, \cdot)=V_{N}\left(x, \xi^{\prime}\right)$ in the notation of Lemma 2.2.

Proof. 1) Construction of $\bar{\nu}_{x}$. We proceed as in the proof of Lemma 2.2. As explained there, we only need to consider the case $C=C^{\prime}=W$. The measure $\bar{\nu}_{x}$ will again be constructed by prescribing its conditional probabilities

$$
q_{x, n}\left(a, a^{\prime}\right)=\bar{\nu}_{x}\left(\xi_{n}=a, \xi_{n}^{\prime}=a^{\prime} \mid\left(\xi_{i}, \xi_{i}^{\prime}\right), i<n\right), \quad a, a^{\prime} \in G .
$$

This prescription will again be different for different periods of time.

Let $\rho_{1}, \ldots, \rho_{k}$ be the stopping times defined in terms of $v$ in the proof of Lemma 2.2 , and let $\rho$ be the smallest even number exceeding $\rho_{k}$. On the set $\{\rho \geq n\}$ we define $q_{x, n}\left(a, a^{\prime}\right)$ as in Lemma 2.2. This means that the coupled random walks $S_{N}$ and $S_{N}^{\prime}+v$ run through $k$ different regimes which enable them to meet, and then (if $\rho_{k}$ is odd) make one step in unison so that they are still equal at the even time $\rho$. After time $\rho$, we introduce a new regime of alternating uncoupling and recoupling. Namely, $S_{N}$ and $S_{N}^{\prime}+v$ are allowed to make independent steps at odd times, and then interchange these steps to meet again at even times. The independent steps at odd times give $V_{N}(x, \cdot)$ and $V_{N}^{\prime}\left(T_{v} x, \cdot\right)+k$ the chance to meet. Once this is achieved, we let $S_{N}$ and $S_{N}^{\prime}+v$ evolve in unison. (The idea to introduce this regime is taken from [2].) 
To make the preceding description precise we introduce the stopping time

$$
\sigma_{x} \equiv \sigma_{x, v, k}=\inf \left\{N>\rho: N \text { even, } V_{N}(x, \cdot)=V_{N}^{\prime}\left(T_{v} x, \cdot\right)+k\right\},
$$

where as usually $\inf \emptyset=\infty$. For each $n$ we require that on the set $\left\{\rho \leq n<\sigma_{x}\right\}$,

$$
q_{x, n}\left(a, a^{\prime}\right)=\left\{\begin{array}{cl}
p(a) p\left(a^{\prime}\right) & \text { if } n \text { is odd } \\
\delta\left(\xi_{n-1}^{\prime}, a\right) \delta\left(\xi_{n-1}, a^{\prime}\right) & \text { if } n \text { is even, }
\end{array}\right.
$$

where $\delta(\cdot, \cdot)$ is the Kronecker-Delta. The final recoupling is achieved by requiring that on $\left\{\sigma_{x}<n\right\}, q_{x, n}\left(a, a^{\prime}\right)=p(a) \delta\left(a, a^{\prime}\right)$.

By the Ionescu-Tulcea theorem, there exists a unique probability measure $\bar{\nu}_{x}$ on $W \times W$ with conditional probabilities $q_{x, n}$, and since these depend measurably on $x$ it follows by routine arguments that $x \rightarrow \bar{\nu}_{x}$ is a probability kernel.

2) Verification of property (i). We consider the conditional two-step probabilities

$$
\bar{\nu}_{x}\left(\xi_{n}=a, \xi_{n}^{\prime}=a^{\prime}, \xi_{n+1}=b, \xi_{n+1}^{\prime}=b^{\prime} \mid\left(\xi_{i}, \xi_{i}^{\prime}\right), i<n\right)
$$

for odd $n$ and $a, a^{\prime}, b, b^{\prime} \in G$. On $\left\{\rho<n \leq \sigma_{x}\right\}$, these are equal to $p(a) p\left(a^{\prime}\right)$ $\delta\left(a^{\prime}, b\right) \delta\left(a, b^{\prime}\right)$, whence

$$
\bar{\nu}_{x}\left(\xi_{n}=a, \xi_{n+1}=b \mid\left(\xi_{i}, \xi_{i}^{\prime}\right), i<n\right)=p(a) p(b)
$$

on this set, and similarly for the primed marginal. In the proof of Lemma 2.2 we have seen that the same conclusion holds on the complementary set. This proves (i).

3) Verification of property (ii). We need to show that $\bar{\nu}_{x}\left(\sigma_{x}<\infty\right)=1$ for $\mu$-almost all $x \in E$. From the proof of Lemma 2.2 we know that $\bar{\nu}_{x}(\rho<\infty)=1$. For fixed $x \in E$ and $v \in \Gamma$ we consider the functions

$$
\begin{aligned}
Z_{N} \equiv Z_{N, x, v} & =\left[V_{N}(x, \cdot)-V_{N \wedge \rho}(x, \cdot)\right]-\left[V_{N}^{\prime}\left(T_{v} x, \cdot\right)-V_{N \wedge \rho}^{\prime}\left(T_{v} x, \cdot\right)\right] \\
& =\sum_{\rho<n \leq N \wedge \sigma_{x}, n \text { odd }}\left[1_{\Lambda}\left(T_{S(n)} x\right)-1_{\Lambda}\left(T_{S^{\prime}(n)+v} x\right)\right]
\end{aligned}
$$

on $W$. [The last equality comes from the fact that $S_{n}=S_{n}^{\prime}+v$ for even $n>\rho$ and all $n>\sigma_{x}$, with $\bar{\nu}_{x}$-probability one.] It can easily be checked that the sequence $\left(Z_{N}\right)$ is a martingale relative to $\bar{\nu}_{x}$. The increments of $\left(Z_{N}\right)$ are \pm 1 or 0 . It follows that $\left(Z_{N}\right)$ either converges or oscillates unboundedly, in that $\sup Z_{N}=\infty$ and $\inf Z_{N}=-\infty$. On the latter event, $\sigma_{x}$ is both infinite and, by a 'continuity' argument, finite. So this event cannot occur with positive probability, and we can conclude that $Z_{N}$ converges with $\bar{\nu}_{x}$-probability one.

To complete the proof of property (ii) it is therefore sufficient to show that $\bar{\nu}_{x}\left(Z_{N}\right.$ converges, $\left.\sigma_{x}=\infty\right)=0$ for $\mu$-almost all $x$. To check this we write for any even $M$ and $u \in G$

$$
\begin{gathered}
\bar{\nu}_{x}\left(Z_{N} \text { converges, } \sigma_{x}=\infty \mid \rho=M, S_{M}=u\right) \\
\leq \quad \bar{\nu}_{x}\left(1_{\Lambda}\left(T_{u+\xi(M+1)+\ldots+\xi(n+1)} x\right)=1_{\Lambda}\left(T_{u+\xi(M+1)+\ldots+\xi(n)+\xi(n+2)} x\right)\right. \\
\text { for all sufficiently large even } \left.n \mid \rho=M, S_{M}=u\right) \\
=\nu\left(1_{\Lambda}\left(T_{S(n)+\xi(n+1)+u} x\right)=1_{\Lambda}\left(T_{S(n)+\xi(n+2)+u} x\right)\right. \\
\text { for all sufficiently large even } n)
\end{gathered}
$$


The inequality follows from the particular definition of $q_{x, n}$ on $\left\{\rho<n \leq \sigma_{x}\right\}$. The equality is a consequence of the fact that the sequence $\left(\xi_{n}\right)_{n>M}$ has distribution $\nu$ and is independent of $\left\{\rho=M, S_{M}=u\right\}$. The subsequent Lemma 3.2 will show that the last probability in (5) vanishes for $\mu$-almost all $x$ and all $u$. So for these $x$ we have

$$
\bar{\nu}_{x}\left(\sigma_{x}<\infty\right) \geq \bar{\nu}_{x}\left(Z_{N} \text { converges }\right)=1
$$

This completes the proof of the lemma.

Lemma 3.2 Under assumptions (A) and (B), the last probability in (5) vanishes for $\mu$-almost all $x \in E$ and all $u \in G$.

Proof. Since $\mu$ is invariant under $T_{u}$, we only need to consider the case $u=0$. The $\mu$-integral of the probability in question is equal to

$$
1-P\left(T^{2 n} \in \Sigma \text { for infinitely many } n\right)
$$

where $\Sigma=\left\{(x, w) \in \Omega: x \in T_{w_{1}}^{-1} \Lambda \triangle T_{w_{2}}^{-1} \Lambda\right\}$. Assumption (B) implies that $P(\Sigma)>0$, and the proposition shows that $T^{2}$ is ergodic. The lemma thus follows from the Poincaré recurrence theorem.

We now turn to the proof of the theorem. In fact we shall prove (3) for all $A \in \mathcal{F}$. As in the proof of the proposition, we can assume that $A=\Delta \times C$ for some $\Delta \in \mathcal{B}$ and a cylinder event $C \subset W$ such that $P(A)=\mu(\Delta) \nu(C)>0$. Let $\bar{\mu}=\bar{\mu}_{\Delta, \Lambda}$ be chosen according to Lemma 2.1, and for $v \in \Gamma$ and $x \in E$ we let $\bar{\nu}_{x, v}=\bar{\nu}_{x, v, 0, C, W}$ be as in Lemma 3.1. We define a probability measure $\bar{P}$ on $\Omega \times \Omega$ by

$$
\bar{P}\left(d x, d x^{\prime}, d w, d w^{\prime}\right)=\bar{\mu}\left(d x, d x^{\prime}\right) \bar{\nu}_{x, \gamma\left(x, x^{\prime}\right)}\left(d w, d w^{\prime}\right) .
$$

Letting again $X, X^{\prime}, \xi, \xi^{\prime}$ denote the four projections on $\Omega \times \Omega$, we then have that $\bar{P}((X, \xi) \in \cdot)=P(\cdot \mid A)$ and $\bar{P}\left(\left(X^{\prime}, \xi^{\prime}\right) \in \cdot\right)=P_{\Lambda}$.

We consider the coupling time

$$
\sigma=\inf \left\{k \geq 1: S_{N}=S_{N}^{\prime}+\gamma\left(X, X^{\prime}\right) \text { and } V_{N}(X, \xi)=V_{N}\left(X^{\prime}, \xi^{\prime}\right) \text { for all } N \geq k\right\} \text {. }
$$

By construction, $\bar{P}(\sigma<\infty)=1$. As in the proof of the proposition we see that, on the set $\{\sigma \leq k\}, \theta^{N} \xi=\theta^{N} \xi^{\prime}$ and $T_{S(N)} X=T_{S^{\prime}(N)} X^{\prime}$ for all $N \geq k$ and, in addition, $V_{N}(X, \xi)=V_{N}\left(X^{\prime}, \xi^{\prime}\right)$ for all $N \geq k$. The last property implies that $\tau_{k}(X, \xi)=$ $\tau_{k}\left(X^{\prime}, \xi^{\prime}\right)$. Hence $T^{\tau_{k}}(X, \xi)=T^{\tau_{k}}\left(X^{\prime}, \xi^{\prime}\right)$ on $\{\sigma \leq k\}$. An estimate analoguous to (4) thus shows that

$$
\left\|P\left(T^{\tau_{k}} \in \cdot \mid A\right)-P_{\Lambda}\right\| \leq 2 \bar{P}(\sigma>k) \rightarrow 0 \text { as } k \rightarrow \infty .
$$

This proves (3) for all $A \in \mathcal{F}$ and in particular the theorem. 


\section{References}

[1] H. O. Georgii: Orbit coupling. Preprint (1995)

[2] W. Th. F. den Hollander: Mixing properties for random walk in random scenery. The Annals of Probab. 16 (1988), 1788-1802.

[3] S. Kakutani: Induced measure preserving transformations. Proc. Japan Acad. Ser. A Math. Sci. 19 (1943), 635-641.

[4] S. Kakutani: Random ergodic theorems and Markoff processes with a stable distribution, in: J. Neyman (ed.), Proc. Second Berkeley Symp. Math. Statist. and Probab.. Univ. of California Press: Berkeley, 1951, pp. 247-261.

[5] P. W. Kasteleyn: Random walks through a stochastic landscape. Bull. Internat. Inst. Statist. 45 (1985), 27-I.1-13.

[6] M. Keane \& W. Th. F. den Hollander: Ergodic properties of color records. Physica 138A (1986), 183-193.

[7] Th. M. Liggett: Interacting Particle Systems. Springer: New York etc., 1985.

[8] I. Meilijson: Mixing properties of a class of skew-products. Israel J. Math. 19 (1974), 266-270. 